\title{
DEMOLITION DUST FORMATION, DIFFUSION MECHANISM AND MONITORING QUANTITATIVE RESEARCH ON DEMOLITION OF EXISTING BUILDINGS
}

\author{
LIU, W. ${ }^{*}$ - TANG, P. T. - LI, K. - JIANG, T. \\ School of Civil Engineering and Architecture, East China Jiaotong University, Nanchang \\ 330013, China \\ *Corresponding author \\ e-mail: liuweijx13@163.com; phone: +86-138-7061-8920 \\ (Received $7^{\text {th }}$ Jun 2019; accepted $10^{\text {th }}$ Oct 2019)
}

\begin{abstract}
By monitoring the change of dust fall concentration at the same height and different distances near the boundary of a building demolition site, this paper tries to study the law of dust formation and diffusion in the area site. In order to conduct quantitative analysis on the raise dust distribution in the construction site at the demolition stage, the amount of fine particulate matter $\mathrm{PM}_{2.5}$ and inhalable particle $\mathrm{PM}_{10}$ in such a site where an experimental demolition is performed measured. The data measurement is carried out with the help of logarithm conversion, so as to reduce the data variability and enable it to approach normal distribution, and the data with the confidence coefficient of $95 \%$ is selected. The main components of $\mathrm{PM}_{2.5}$ and $\mathrm{PM}_{10}$ emissions are extracted to indicate the emission intensity. According to the emission intensity, a construction site can be divided into three categories for analysis by hierarchical clustering, so as to obtain the desired results. Through the comparison and analysis of the monitoring quantitative and discrete phase model, the motion, diffusion and settlement equations of the dust of the demolition site are verified, indicating that the simulation results using the discrete phase model are credible.
\end{abstract}

Keywords: raise dust in construction, formation mechanism, discrete phase model, diffusion mechanism, monitoring quantification

\section{Introduction}

In recent years, the value orientation of the public policy making in China has gradually transformed from emphasizing the pursuit of economic efficiency to focusing more on social equity, with an expectation of balancing the, dual goals of economic development and social welfare. With the acceleration of the urbanization process, the demolition of old buildings is increasing day by day. As a way to the old buildings in an unprecedented manner, the building demolition project is also included in one of the important tasks of the construction project. The demolition of old buildings has produced a lot of construction waste. "The amount of construction waste in China already accounts for 30 to $40 \%$ of the total amount of urban waste," said Qiu Baoxing, a former vice-minister of housing and urban-rural development. According to the rough statistics of the loss of construction materials of brick and concrete structure, whole cast-in-situ structure and frame structure, in the construction process of every $10,000 \mathrm{~m}^{2}$ of buildings, only the construction waste will produce 500 to 600 tons; Every 10,000 $\mathrm{m}^{2}$ of old buildings demolished will generate 7,000 to 12,000 tons of construction waste, and $40 \%$ of the old buildings demolished in China each year. Wang Guangtao, former minister of construction, said that by 2020 , China will add 30 billion $\mathrm{m}^{2}$ of construction area, and the total amount of construction waste will be 1.8 billion tons. This poses a huge environmental threat to China. These construction units or individuals usually do not carry out any treatment on the muck, spoil, waste, mud and other waste generated in 
the demolition of various buildings, structures and pipe networks. Moreover, the raise dust, sand dust and other problems generated in waste transportation and stacking have caused serious environmental pollution. Raise dust can be hazardous human health and it is likely to cause dust pollution, which in turn leads to deterioration of the surrounding environment. Studies have shown that air pollution can be divided into two types: gaseous pollution and particulate pollution. Particles with a diameter of less than $10 \mu \mathrm{m}$ are called inhalable particles $\left(\mathrm{PM}_{10}\right)$, which are rather harmful to human health. Inhalable particles can be divided into fine particles $\left(\mathrm{PM}_{2.5}\right)$ and coarse particles according to the particle size. In recent years, the $\mathrm{PM}_{2.5}$ index has attracted a lot of people's attention. The main reason is that fine particles (also known as microparticles) can directly enter and precipitate in human lungs. Its impact on human health is permanent. If a large amount of building raise dust enters the lung, it will cause obstructive damage to the respiratory system, reduce the ventilatory function of the bronchus, and lose the air exchange function the alveoli. In the meanwhile, fine particles adsorbed in the lungs stimulate the cell wall, causing bronchitis, asthma and other diseases. In addition, the increased concentration of $\mathrm{PM}_{10}$ and $\mathrm{PM}_{2.5}$ in the atmosphere will lead to cardiovascular disease, resulting in an increase in the incidence rate of cancer. The increase of particles in the air easily leads to the appearance of haze weather condition and affects people's normal travel. Acidic substances in the air have a certain corrosive effect on buildings. If the amount of acidic substances increases, the corrosion degree is improved, and the hazard is rather serious especially after rainfall. Therefore, the research on the generation, movement law and concentration of raise dust in the demolition construction site of existing buildings is the basis for the construction site employees to make preparations and control in advance, an important means to protect the health and life of employees, and an important prerequisite for ecological environmental protection.

For many years, various researches have been carried out on raise dust pollution in building construction at home and abroad, including the exploration of raise dust emission factors for building construction (Muleski et al., 2005; Toropov et al., 2018). Raise dust is an open pollution source entering the atmosphere and it is an important component of total suspended particulates in the ambient air. According to the dust research, the causes of dust mainly come down to the following two aspects: The powder is separated from the powder flow because of the influence of the surrounding air during the falling process, and it suspends in the transition layer of the powder flow (Ansart et al., 2009), thus generating the dust cloud. The falling powder particles collide with particles and walls in the bag to produce impact dust and suspend in the air (Wang et al., 2014). The essence of the phenomenon that powders take up air and the air affects particles is gas-solid collaboration effect (Alekhfne, 2012; Cerecedo et al., 2009). Fan et al. (2019) conducted accumulated dust load sampling and laboratory screening analysis on typical roads, measured the road dust particle size distribution and particle size multiplier by resuspension system and particle size spectrometer, investigated the road vehicle composition and calculated the vehicle weight, and applied the model method to establish a localized road raise dust $\mathrm{PM}_{2.5}$ emission factor. Based on the survey data of architecture and municipal construction projects in Xi'an, Xiao et al. (2019) combined with two sets of emission factors of typical cities in northern China measured by different institutions to estimate the emission amount and emission strength of the architecture construction raise dust $\mathrm{PM}_{10}$ and $\mathrm{PM}_{2.5}$ in $\mathrm{Xi}^{\prime}$ an in 2017 , so as to construct a list of particulate matter emissions from county-level buildings in $\mathrm{Xi}$ 'an, and analyze 
the spatial distribution characteristics. All local governments have attached great importance to this and have issued a series of guidelines and policies to monitor the raise dust pollution. However, the current construction raise dust pollution monitoring still relies on manual patrol and fixed-point monitoring, which is inefficient and ineffective, and there is no more effective monitoring techniques. The construction environment monitoring mainly focuses on the raise dust and material waste and so on at the construction site (Kohlman et al., 2014), in which, the construction raise dust control is a research hotspot. In terms of monitoring methods, instrument sampling is the main method and video monitoring is feasible as well (Wu et al., 2015). However, although the above methods have high precision, they are not efficient because of being easily interfered. In recent years, UAV (Unmanned Aerial Vehicle) images have been used for water pollution monitoring, chemical gas pollution monitoring, vehicle identification, building deformation monitoring and so on (Chen et al., 2016). Construction dust is the main source of atmospheric pollutants and the key object of construction environment monitoring, but the traditional monitoring methods have limited efficiency and effectiveness. For this purpose, Ma et al. (2018) integrated the use of UAV and image recognition technology, designed the structure, function and operation process of the construction dust pollution source automatic monitoring system with HSV (Hue, Saturation, Value) feature extraction, histogram comparison, nonzero pixel calculation as the core, and tested the operation test. It is found that the pollution source characteristic comparison effect is relatively stable, basically meeting the construction dust pollution source monitoring demand Wu et al. (2017) designed a construction site environmental monitoring system to achieve real-time monitoring of the construction site environment. In order to propose a targeted construction dust prevention and control measure, ensure the occupational health and safety of construction workers, Li et al. (2014) selected two main residential projects in Beijing and arranged the raise dust concentration monitoring sites in the main construction activity areas at the second structure and indoor rough decoration stages to analyze the emission characteristics and main distribution laws of raise dust. Huang et al. (2013) considered that the screening of dust source samples is an elementary test for studying the dust pollution characteristics. It has a wide application. The screen test is required whether it is to determine the critical friction wind speed of wind erosion dust, road dust load and other parameters, or to prepare a dust source resuspension test sample for studying the particle size distribution and source composition spectrum of total suspended particulate matter. Shi et al. (2013) applied laser demand technology to find that the non-structured particles in the near-surface atmosphere have important theoretical and practical values. The laser demand technology is used to detect the raise dust of urban construction sites, and the outer wall of the building is utilized to conduct relatively calibration of the laser demand signal. Afterwards, according to the laser demand equation, the extinction coefficient of the raise dust particles along the optical path is solved, and the extinction coefficient distributions of multiple paths are obtained through the process control scanning station. In the end, the two-dimensional mass concentration distribution condition is achieved by combining with the mass extinction efficiency factor.

Dong et al. (2019) such as using the positive definite matrix factorization model (PMF) - the health risk assessment model (HMHR) explores the Yangzhou fine particulate matter (PM2.5) in heavy metal pollution sources and little pollution, and combine the health risk assessment model (heavy metal health risk, HMHR) to calculate 
the influence of different pollution on human health, to reveal the different sources of pollution potential harm to human body health to provide scientific information. Atmospheric fine particulate matter (PM2.5) (aerodynamic equivalent diameter $<2.5$ micron) has an impact on human health and environmental air quality. Among them, heavy metals have a particularly significant impact on human health, which can enter the human body through hand ingestion, breathing and skin contact, and further lead to functional dysfunction and irreversible injury. Elements $\mathrm{Cr}$ and $\mathrm{Pb}$ are elements with the highest potential health risks in cities, which can cause diseases of the nervous system and blood system, especially for infants and young children (Thomas et al., 2009). PM2.5 receptor samples were collected, positive matrix factorization (PMF) was used to analyze the contribution of different pollution sources to PM2.5 and different heavy metals, and the heavy metal health risk HMHR was combined to calculate the impact of different pollution sources on human health (Peng et al., 2016). Li et al. (2015) evaluated the pollution of construction workers by dust at different construction stages by establishing a model of health damage caused by construction dust. Chou et al. (2015) calculated the economic loss caused by CO2 emission to construction workers in the construction process by establishing Monte Carlo simulation model. However, most of these studies only focus on the occupational health damage to construction workers, which to some extent cannot accurately reflect the impact scope and degree of construction dust.

Sung et al. (2006) observed that building demolition was one of the most common activities in the construction industry. The commonly used demolition techniques are mechanical demolition, demolition and mixed demolition. Although deconstruction is advocated in an environmentally friendly way, the cost comparison of demolition projects with different technologies is rarely studied. Mohamed et al. (2008) proposed a new framework for building pollution control based on multi-objective optimization. The method used in the proposed framework is based on the calculation of pollution from dust, hazardous gases and noise to each activity involved in the project. Madelyn et al. (2010) proposed a new method based on the building cost system (BCS) to deal with the costs associated with construction and demolition waste management, which improves accuracy and produces estimates independent of the general budget. Water suppression to mitigate the spread of dust is the most popular and a universal method to deal with construction dust (Zuo et al., 2017). However, countries or regions which experience water shortage find this technique to be expensive and morally incorrect. Maintaining standard heights for stockpiling, wetting objects before cutting or grinding, wetting while sweeping are very easy methods to control some of the dust generated at sites. Use of chemical agents, electrical sweepers, dust screens, local exhaust ventilation, etc., are some technologies that are available for the construction companies to manage dust pollution (Wu et al., 2016).

At present, China has carried out rather sufficient research on the raise dust pollution in construction projects and storage yards, but there is indeed insufficient research on raise dust in demolition projects. The raise dust emission law of the demolition project is rather complicated. Up to now, China has not published any feasible management method with the emission limit as the basis, which can be used to quantitatively evaluate the raise dust emission strength of the demolition projects. Although some provinces and municipalities have published a series of prescribed measures for the raise dust of demolition projects and put forward corresponding controls to the generated raise dust in heavy demolition area, most of these regulations are behavioral 
regulations, which are lack of quantitative understanding and control, and researches on the emission characteristics of demolition raise dust pollution, so it is difficult to accurately grasp the emission intensity of dust emission in demolition projects. This study takes the formation and diffusion of raise dust in the demolition process of existing buildings as the research object, explores the relevant laws of dust formation and diffusion, regards green construction environmental protection as the objective, provides theoretical and practical basis for the raise dust monitoring quantification of the construction site. Based on the gas-solid two-phase flow theory, the method of simulation, monitoring and quantification of the movement, diffusion and settlement equation of demolition construction dust is used to study the formation and diffusion of dust in the demolition construction.

\section{Methods}

\section{Variable selection and model establishment}

\section{Confirmation of raise dust formation and diffusion impact factor}

This research selects the falling dust as the inspection index of analyzing the laws of raise dust formation and diffusion. Falling dust refers to particulates that naturally settle to the surface by gravity under airborne conditions. The amount of dust generated in the demolition construction is not only related to the construction stage and construction strength factors, but also has a certain correlation with the climatic conditions in demolishing the building. Through literature research and consultation with experts, it has been determined that the raise dust formation and diffusion impact factors include: dust particle diameter, gas density, dust particle density, dust moisture content, construction strength, wind speed, temperature, humidity, height and other factors.

\section{Equation of motion of the dust in the air}

The dust unmooring load refers to the force overcoming the gravity of the dust particles, the gravitation and adhesion force among particles to fly the dust. The critical load that causes the dust to rise is called the ultimate unmooring force. However, according to Zhi et al. (2018), the VWD (Visual Web Developer) force is used to calculate the gravitational attraction between dust particles, which is negligible under normal conditions. In studying the dust in this paper, it is assumed to be a spherical particle. The stress of the dust is very complicated at the construction site. The force of the dust particles in demolishing the existing buildings is analyzed, and the dust is found to be affected by gravity, buoyancy force, air resistance and other external forces. The resultant force $\mathrm{F}_{\alpha 0}$ of the dust is determined by the following equation:

$$
F_{a 0}=F_{\mathrm{g}}+F_{f}+F_{d}+F_{u n}
$$

In the equation, $F_{\alpha 0}$ is the resultant force of the dust, with the unit of $N . F_{g}$ is the weight of the powder particle, with the unit of $N . F_{f}$ is the adhesion force among powder particles, with the unit of $N . F_{d}$ is the air resistance of the dust particles, with the unit of $N$. $F_{\text {un }}$ is other forces of the dust particles, with the unit of $N$.

The movement law of dust in the air essentially belongs to the research area of gassolid two-phase flow (Zhu et al., 2017). The volume of dust particles is much smaller than the volume of air. In order to simplify the equation of motion of dust at the 
construction site, the volume occupied by dust particles can be neglected. In gas-solid two-phase flow, solid particles are exerted with more and complex forces. For the convenience of calculation, it is assumed that the dust is not affected by other forces, but only the force caused by the relative motion of the gas-solid two phases, the equation of motion of the dust is established in Cartesian coordinates according to Newton's second law (Sun et al., 2016):

$$
m_{\mathrm{p}} \frac{d \nu_{p}}{d t}=\frac{\pi}{6} d_{p}^{3} \rho_{p} g+\frac{\pi}{6} d_{p}^{3} \rho_{8} g+\frac{\pi \mathrm{C}_{p}^{2} \boldsymbol{\nu}_{p}^{2} \rho_{p}}{8}
$$

After simplification, it can be expressed as:

$$
\frac{d v_{p}}{d t}=\frac{3 C_{d} \rho_{g}\left(v_{g}-v_{p}\right)^{2}}{4 d_{p} \rho_{p}}
$$

In the equation: $m_{p}$ is the dust mass, with the unit of $\mathrm{mg} ; v_{p}$ is the dust movement speed, with the unit of $\mathrm{m} / \mathrm{s} ; t$ is the movement time, with the unit of $s ; d_{p}$ is the dust particle diameter, with the unit of $\mathrm{m} ; \rho_{P}$ is the dust particle density, with the unit of $\mathrm{kg} \cdot \mathrm{m}^{-3} ; g$ is the gravitational acceleration, with the unit of $\mathrm{m} / \mathrm{s}^{2} ; \rho_{g}$ is the gas density, with the unit of $\mathrm{kg} \cdot \mathrm{m}^{-3} ; C_{d}$ is the air resistance coefficient; $v_{p}$ is the moving speed of the dust particles, with the unit of $\mathrm{m} \cdot \mathrm{s}^{-1} ; v_{g}$ is the gas movement speed, with the unit of $\mathrm{m} \cdot \mathrm{s}^{-1} ; \pi$ is valued as 3.14 .

\section{Diffusion equation of dust in air}

The dust particles with smaller particle diameter generated in the demolition process of the existing building have small gravity and low sedimentation speed. Due to the impact of the Brownian motion of the air molecules, irregular movements are generated accordingly. The research shows that the smaller the particle size of the dust particles, the more intense the dust particles move. This movement is named as the dust diffusion movement.

Any particle fluid unit in the space (as shown in Fig. 1) is used to analyze the dust diffusion motion ( $\mathrm{Li}$ et al., 2006), assuming that the volume of the particle unit is $\Delta V=\Delta x \cdot \Delta y \cdot \Delta z$. The mass flux of a substance (called as flux for short) refers to the mass of a substance that flows through.

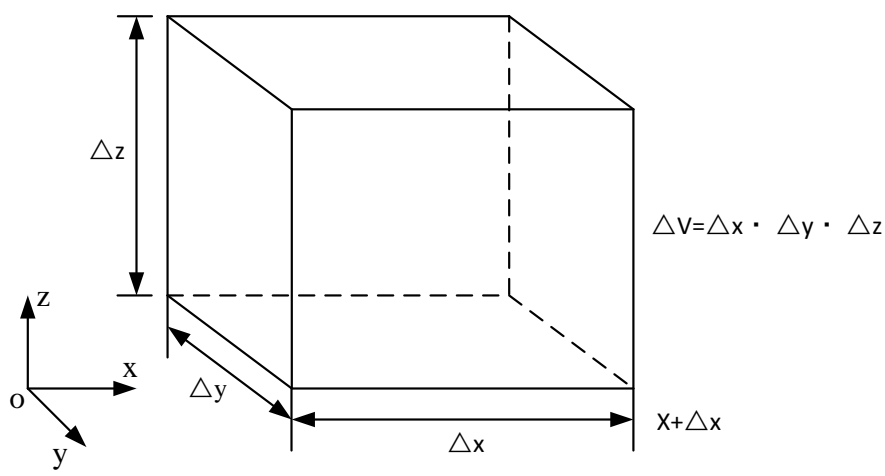

Figure 1. Diagram of dust diffusion model 
According to the gradient conveying theory, the amount of dust passing through a unit area along the $\mathrm{x}$ direction for the dust unit time suspended in the air is proportional to the dust mass concentration gradient in the $\mathrm{x}$ direction. The flux of dust in the $\mathrm{x}$ direction is:

$$
\mathrm{Q}_{\mathrm{x}}=-K_{\mathrm{x}} \frac{\partial c}{\partial x}
$$

In the equation: $Q_{x}$ is the flux of dust in the $x$ direction, with the unit of $\mathrm{mg} \cdot \mathrm{m}^{-3} \cdot \mathrm{s}^{-1} ; K_{x}$ is the diffusion coefficient of dust in the x direction; $c$ is the mass concentration of dust, with the unit of $\mathrm{mg} \cdot \mathrm{m}^{-3} ; \frac{\partial c}{\partial x}$ is the dust mass concentration gradient along the $x$ direction.

Under the action of the wind flow at the construction site, the dust particles will move along the wind flow direction under the action of the wind flow. Combined with the diffusion action of the dust, the dust concentration in the wind flow movement direction will keep on changing. The wind speeds in the $x, y$, and $z$ directions are set as $v_{x}, v_{y}$, and $v_{z}$, respectively. According to the mass conservation equation, the net inflow in three directions is equal to the cumulative rate, then:

$$
\frac{\partial c}{\partial \mathrm{t}}=-v_{\times} \frac{\partial c}{\partial x}-v_{\mathrm{y}} \frac{\partial c}{\partial \mathrm{y}}-v_{\mathrm{z}} \frac{\partial c}{\partial \mathrm{z}}+K_{\times} \frac{\partial^{2} c}{\partial x^{2}}+K_{\mathrm{y}} \frac{\partial^{2} c}{\partial \mathrm{y}^{2}}+K_{\mathrm{z}} \frac{\partial^{2} c}{\partial \mathrm{z}^{2}}
$$

In practical applications, in order to simplify the calculation, a one-dimensional stationary diffusion equation can be selected for the research in the three-dimensional coordinate system. When the $\mathrm{x}$-axis direction in the three-dimensional coordinate system is selected for the calculation, $v_{y}=v_{z}=0$. The above equation (Eq. 5) can be simplified as:

$$
\frac{\partial c}{\partial \mathrm{t}}+v_{\times} \frac{\partial c}{\partial x}=K_{\times} \frac{\partial^{2} c}{\partial x^{2}}+K_{\mathrm{y}} \frac{\partial^{2} c}{\partial \mathrm{y}^{2}}+K_{\mathrm{z}} \frac{\partial^{2} c}{\partial \mathrm{z}^{2}}
$$

When the wind speed and turbulence intensity are in a steady state, $\frac{\partial c}{\partial \mathrm{t}}=0$. When the ambient wind speed is high, the diffusing capacity of the dust in the $x$ direction $K_{\times} \frac{\partial^{2} c}{\partial x^{2}}$ can be negligible. In such condition, the above equation $(E q .6)$ can be further simplified as:

$$
\nu_{\mathrm{x}} \frac{\partial c}{\partial x}=K_{\mathrm{y}} \frac{\partial^{2} c}{\partial \mathrm{y}^{2}}+K_{\mathrm{z}} \frac{\partial^{2} c}{\partial \mathrm{z}^{2}}
$$

The diffusion coefficients of the dust particles with the same property in the $x, y$, and $z$ directions are equal, which are uniformly expressed by $K_{p}$ (Chen et al., 2015). The 
differential equation ( $E q .7)$ is solved by Gauss formula, so as to obtain the diffusion equation of dust in air as follows:

$$
c=k x^{-1} \exp \left[-\frac{v_{\mathrm{x}}}{4 K_{\mathrm{p}} x}\left(y^{2}+z^{2}\right)\right]
$$

In the equation, $k$ is the Boltzmann constant, which is valued as $1.38 \times 10^{-23} \mathrm{~J} \cdot \mathrm{K}^{-1}$.

\section{Sedimentation equation of the dust in the air}

Dust particles settle under gravity during the process of demolishing existing buildings. During the sedimentation process, the particle velocity changes continuously, and the resistance transforms accordingly. When the gravity, buoyancy and air resistance reach a balance, the dust particles settle at a constant speed, and the sedimentation velocity also reaches a maximum value at this moment. Such a speed is called Stokes velocity.

When the dust settles gradually, it is in the stress equilibrium status, and it is $F=F_{g^{-}}$ $F_{f}=F_{d}$ at this moment. Hence, the following equation is obtained:

$$
\frac{\pi}{6} d_{p}^{3}\left(\rho_{p}-\rho_{g}\right) g=\frac{\pi \mathrm{C} d_{p}^{2} v^{2} \rho_{p}}{8}
$$

When the dust begins to settle evenly, the sedimentation velocity also reaches a maximum value, that is, $v=v_{t}$. The dust sedimentation speed is as follows:

$$
v_{\mathrm{t}}=\sqrt{\frac{4\left(\rho_{p}-\rho_{g}\right) g d_{p}}{3 \rho_{s} C_{d}}}
$$

(a) When $R e_{p} \leq 1$, the movement of the dust particles is in a laminar flow state, $C_{d}=\frac{24}{\operatorname{Re}_{p}}$. (b) When $1<R e_{p}<500$, the movement of the dust particles belongs to the turbulent transition state. (c) When $500<R e_{p}<2 \times 10^{5}$, the movement of dust particles belongs to the turbulent state, and $C_{d}$ hardly changes with $R e_{p}$, which is approximately $C_{d} \approx 0.44$. The Reynolds number of the dust particles $R e_{p}$ is a dimensionless number reflecting the relationship between the inertial force and the viscous force in fluid flowing process. Its value is related to the flow velocity, feature size and fluid viscosity. Reynolds number $R e_{p}=\frac{d_{p} \rho_{g} v}{\mu_{\mathrm{g}}}$. In the formula, $\mu_{g}$ is the dynamic viscosity of air, with the unit of $P a \cdot s$.

\section{Empirical analysis}

\section{Profile of demolition project}

The demolished buildings in the demolition project include 107, 107A, 107C, 107D buildings and some demolition projects above the ground surface, with a total floor area of about $5000 \mathrm{~m}^{2}$ and a total construction area of about $13798.95 \mathrm{~m}^{2}$. Among them, 107 
is a teaching building with five-story frame structure, 107A is a hospital with two-story brick-and-mortar structure, $107 \mathrm{C}$ is a dormitory building with four-story frame structure, and 107D is an auditorium with two-story brick-concrete. The demolition of the building is required to be completed in September 11, 2018 October 5, 2018. The construction site is completely enclosed by iron sheets, with a height of no less than $1.8 \mathrm{~m}$. The safe distance of the building enclosing structure away from the building is 1.5 times of the building height, with its east side connected with the wall (refer to Figure 2: construction site planar graph for the building enclosing structure).

\section{Monitoring quantification method}

The research results show that particles with a particle size of less than $50 \mu \mathrm{m}$ will float in the atmosphere for a long time because of the buoyancy and eddy functions, and will be transported to a distant place. Particles with a particle size of 50-100 $\mu \mathrm{m}$ can be kept in suspension, but settle down soon. Particles with a particle size larger than $100 \mu \mathrm{m}$ can only roll on the ground under the action of wind.

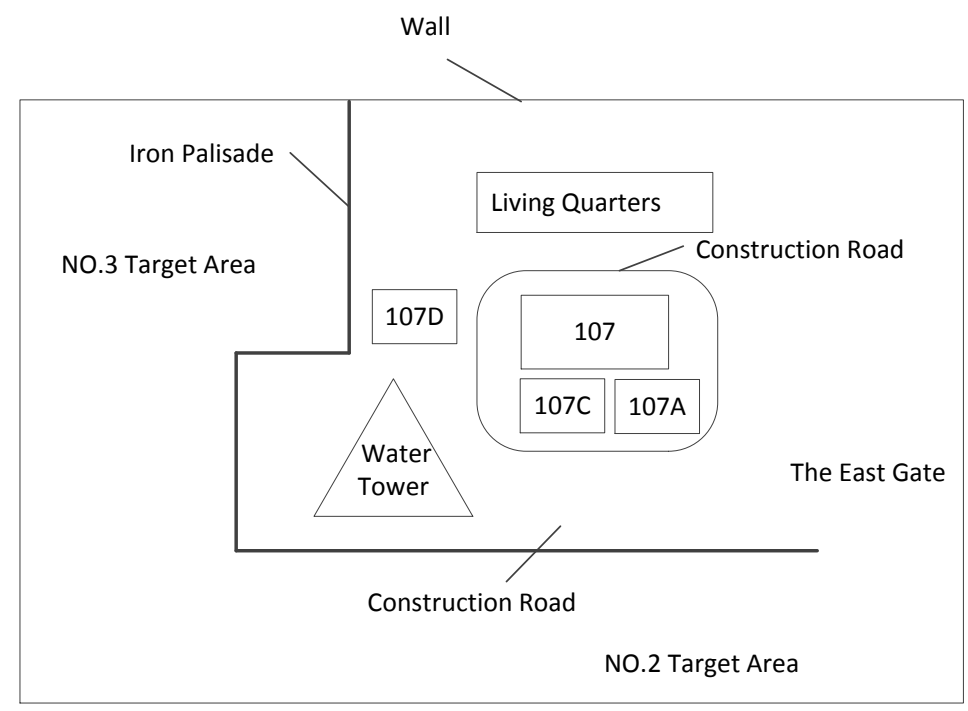

Figure 2. Construction site planar graph

In this research, monitoring is carried out by combining dust removal cylinder collection and weighing and $\mathrm{PM}_{2.5}$ automatic detector. The dust removal cylinder is mainly located at the boundary of the construction site, and the height is no less than $3 \mathrm{~m}$. The particles collected in the dust removal cylinder have a diameter of below $100 \mu \mathrm{m}$, mainly 50-100 $\mu \mathrm{m}$ particles. The instrument for measuring the concentration of particles adopts Xinsite handheld $\mathrm{PM}_{2.5}$ laser dust concentration detector, with the model of HT-9600. The instrument employs the $\mathrm{PM}_{2.5}$ photoelectric sensor principle, and the pumping sampling method is more accurate. The measuring height is about $1.5 \mathrm{~m}$ and the measuring time of each measuring point is $50 \mathrm{~s}$. At the demolition stage, each measurement point shall be recorded of the reading for every $1 \mathrm{~h}$. The monitored data includes: temperature, relative humidity, $\mathrm{PM}_{2.5}$ concentration, $\mathrm{PM}_{10}$ concentration, dust particle size $(0.3 \mu \mathrm{m}, 2.5 \mu \mathrm{m}, 10 \mu \mathrm{m})$ and time record. The hourly mean value of $\mathrm{PM}_{2.5}$ and $\mathrm{PM}_{10}$ measured by the air quality real-time monitoring station at the location 
of building demolition was selected as the background value. This study only conducted raise dust monitoring to the demolition project of 107 teaching building. 16 measuring points were selected around the 107 teaching building, and 16 dust removal cylinders were placed in the measuring points to collect dust. The position of the measuring point is shown in Figure 3. According to the measuring point arrangement method in the similar literature, the wind speed measuring points and the dust mass concentration measuring points were respectively arranged at the demolition site, which were arranged with a certain interval along the wind flow direction. The wind speed measuring points were arranged to have a space of $20 \mathrm{~m}$, and a total of 7 measuring points were arranged. Each measurement point was conducted with the data measurement of no less than 3 times, and then an average value was calculated.

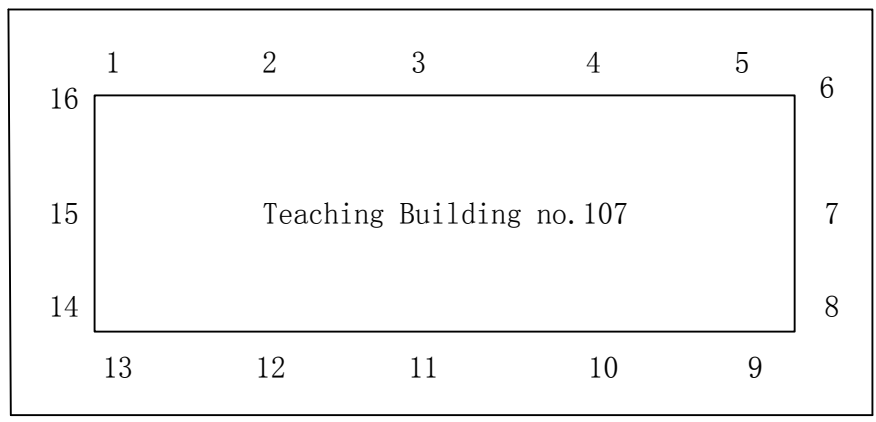

Figure 3. Measuring point position diagram

\section{Data preprocessing}

SPSS22.0 and Excel are used to process the data, logarithmically transform the obtained $\mathrm{PM}_{2.5}$ and $\mathrm{PM}_{10}$ data, so as to reduce the data variability of the original data, and enable the data distribution to approach the normal distribution. Cases with a confidence coefficient of $95 \%$ were selected for the analysis, namely, cases with the data exceeding $(\mu-1.96 \sigma, \mu+1.96 \sigma)$ were eliminated $(\mu$ and $\sigma$ are the mean and standard values of the normal distribution, respectively). Afterwards, the original data and the logarithmic data are analyzed, and the inspection level a is valued as 0.05. Through conducting correlation analysis to the two groups of data after logarithmic transformation, it shows that the Pearson correlation coefficients of the data after the logarithmic transformation and the original data are 0.898 and 0.941 , respectively, presenting an extremely strong correlation. In other words, the logarithmic transformation process is reasonable. 325 measured data is obtained during the demolition phase, and 296 is selected, showing a data efficiency of $91.08 \%$. After the completion of the demolition phase, there is a total of 120 measured data, in which, 112 is selected, showing a data efficiency of $93 \%$. The statistical description of the cases after screening is shown in Table 1 .

\section{Quantitative data analysis}

\section{Emission intensity index}

Correlation analysis is conducted to $\mathrm{PM}_{2.5}$ and $\mathrm{PM}_{10}$ emissions. The Pearson correlation coefficient of the two variables is obtained through analysis: 0.950 , showing that the two variables have an extremely strong correlation. The principal component 
analysis can be used to extract a principal component to achieve variable dimension reduction. Refer to Table 2 for the index characteristic values and contribution rates of the component extraction process obtained by principal component analysis. The factor load matrix is shown in Table 3.

Table 1. Case selection and statistical description

\begin{tabular}{|c|c|c|c|c|}
\hline \multirow{2}{*}{ Category } & \multicolumn{2}{|c|}{ Demolition stage } & \multicolumn{2}{|c|}{ After completing the staged work of demolition } \\
\hline & $\mathrm{PM}_{2.5} /\left(\mu \mathrm{g} \cdot \mathrm{m}^{-3}\right)$ & $\mathbf{P M}_{10} /\left(\mu \mathrm{g} \cdot \mathbf{m}^{-3}\right)$ & $\mathbf{P M}_{2.5} /\left(\mu \mathrm{g} \cdot \mathrm{m}^{-3}\right)$ & $\mathbf{P M}_{10} /\left(\mu \mathrm{g} \cdot \mathrm{m}^{-3}\right)$ \\
\hline Minimum & 10.18 & 49.36 & 10.01 & 28.91 \\
\hline Maximum & 432.30 & 495.40 & 189.24 & 202.20 \\
\hline Mean & 94.409 & 156.521 & 59.511 & 92.881 \\
\hline Standard deviation & 60.573 & 81.404 & 33.220 & 35.444 \\
\hline
\end{tabular}

Table 2. Index characteristic values and contribution rates

\begin{tabular}{c|c|c|c}
\hline Times & Initial characteristic value & Variance percentage /\% & Accumulated variance percentage \\
\hline 1 & 1.904 & 95.189 & 95.189 \\
2 & 0.096 & 4.811 & 100 \\
\hline
\end{tabular}

Table 3. Factor load matrix

\begin{tabular}{c|c}
\hline Project & Principal component 1 load \\
\hline $\mathrm{PM}_{2.5}$ emission & 0.955 \\
$\mathrm{PM}_{10}$ emission & 0.955 \\
\hline
\end{tabular}

It can be seen from Table 2 that the contribution rate of the first principal component extracted is $95.189 \%$. This principal component is selected to reflect the emission intensity of $\mathrm{PM}_{2.5}$ and $\mathrm{PM}_{10}$ on the site. If it is recorded as $Z$, the emission intensity $Z$ can be prepared combined with the component matrix:

$$
\mathrm{Z}=0.512 \times \Delta \mathrm{PM}_{2.5}+0.512 \times \Delta \mathrm{PM}_{10}
$$

The principal component values of each measured case can be calculated according to the principal component equation. Refer to Table 4 for the average principal component value of each measured position.

Table 4. The average principal component value of each measured position

\begin{tabular}{c|c|c|c}
\hline Location & $\begin{array}{c}\text { Average principal } \\
\text { component value }\end{array}$ & Location & $\begin{array}{c}\text { Average principal } \\
\text { component value }\end{array}$ \\
\hline Road side & 69.854 & Living quarter of the workers & 59.003 \\
Waste recycling area & 77.315 & Gunnery area & 145.951 \\
Working passage & 227.444 & Out of the construction wall & 48.080 \\
Excavator & 159.201 & Loading vehicle & 109.694 \\
Plane transport corridor & 91.343 & Construction waste dump & 133.256 \\
\hline
\end{tabular}


In order to identify the location of raise dust emissions to be concentrated during the construction process, and understand the sources and influencing factors of dust at different locations, the locations are classified according to emission intensity. The SPSS self-contained system clustering method is used to classify the locations. After standardizing the emission intensity, the square Euclidean distance is used for classification, so as to draw the system clustering spectrum shown in Figure 4.

According to the diagram, the sixteen positions are divided into three categories: the first category is the outer wall of the construction site, and the average value of the main component $Z$ is 10.416 , indicating that the dust amount at this position is only slightly higher than the background value. It shows that the construction wall has a good restraining effect on dust diffusion. The second category includes the plane transport corridors, the living quarter of the workers, road side, waste recycling area, and around the load vehicle. The third category includes the construction waste dump, gunnery area, excavator area, and working passage. These positions have the highest raise dust emissions and the strongest construction intensity on the construction site. An independent sample $\mathrm{T}$ test is performed on the classification results, obtaining a T-test $\mathrm{P}$-value matrix among the three categories as shown in Table 5.

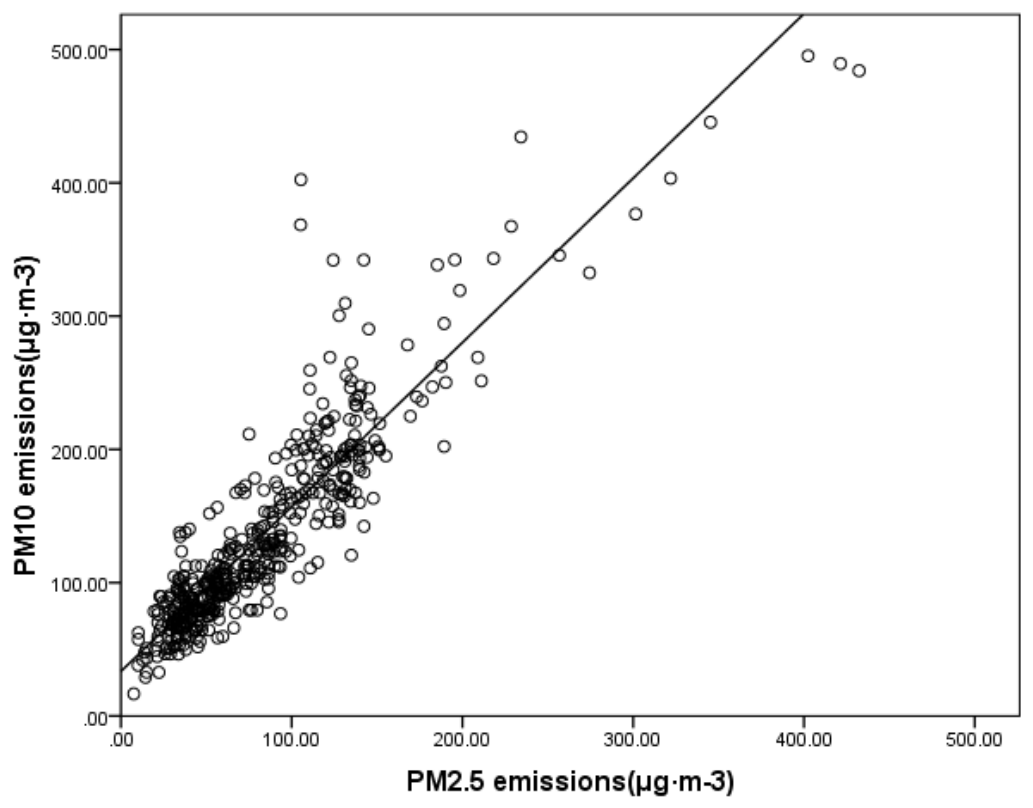

Figure 4. Linear fitting of $P M_{10}$ and $P M_{2.5}$ emission

Table 5. Independent sample T test on the classified result

\begin{tabular}{c|c|c|c}
\hline T test & The first category & The second category & The third category \\
\hline The first category & 1 & 0.019 & $9.5 \times 10^{-5}$ \\
The second category & 0.019 & 1 & $2.4 \times 10^{-5}$ \\
The third category & $9.5 \times 10^{-5}$ & $2.4 \times 10^{-5}$ & 1 \\
\hline
\end{tabular}

According to the test standard $\mathrm{P}<0.05$, it can be seen that the classification of the measuring points into three categories has a good effect. 


\section{$P M_{2.5}$ and $P M_{10}$ emission correlation}

A linear fitting is conducted to $\mathrm{PM}_{10}$ and $\mathrm{PM}_{2.5}$ emissions of each region. The $\mathrm{R}^{2}$ fitting between two types of particle emissions in the region of the first category is 0.806 , while the $\mathrm{R}^{2}$ of linear fitting in the region of the second category is 0.481 . The two has a good linear relationship in the third region with the highest emission intensity.

The linear fitting of $\mathrm{PM}_{10}$ and $\mathrm{PM}_{2.5}$ emissions is shown in Figure 5, with a confidence coefficient of $\mathrm{R}^{2}=0.817$. The following equation can be obtained for linear fitting:

$$
\Delta \mathrm{PM}_{10}=1.234 \times \Delta \mathrm{PM}_{2.5}+33.496
$$

It can be seen from the fitting equation $\mathrm{R}^{2}$ that the linear relationship between the two variables is good, indicating that the $\mathrm{PM}_{2.5}$ and $\mathrm{PM}_{10}$ emissions have the same emission source in the region of the third category. The paired sample $\mathrm{T}$ test is used to test the two emissions, thus obtaining the significance coefficient $4.1^{-12}<0.05$, that is, the $\mathrm{PM}_{10}$ emission is significantly larger than that of $\mathrm{PM}_{2.5}$ in the demolition stage, and the particles discharged are mainly larger than $10 \mu \mathrm{m}$. When the demolition machinery in the region of the third category is working, the construction dust will be generated in the construction area, and the measured dust produces a large amount of $\mathrm{PM}_{2.5}$ and $\mathrm{PM}_{10}$. An independent sample $\mathrm{T}$ test is performed on the emission intensity in doing the demolition work and completing the demolition staged work, obtaining the doubletailed significance coefficient between the two categories is $0.025<0.05$. In other words, the dust emission in the demolition process is significantly higher the dust emission after completing the demolition staged work.

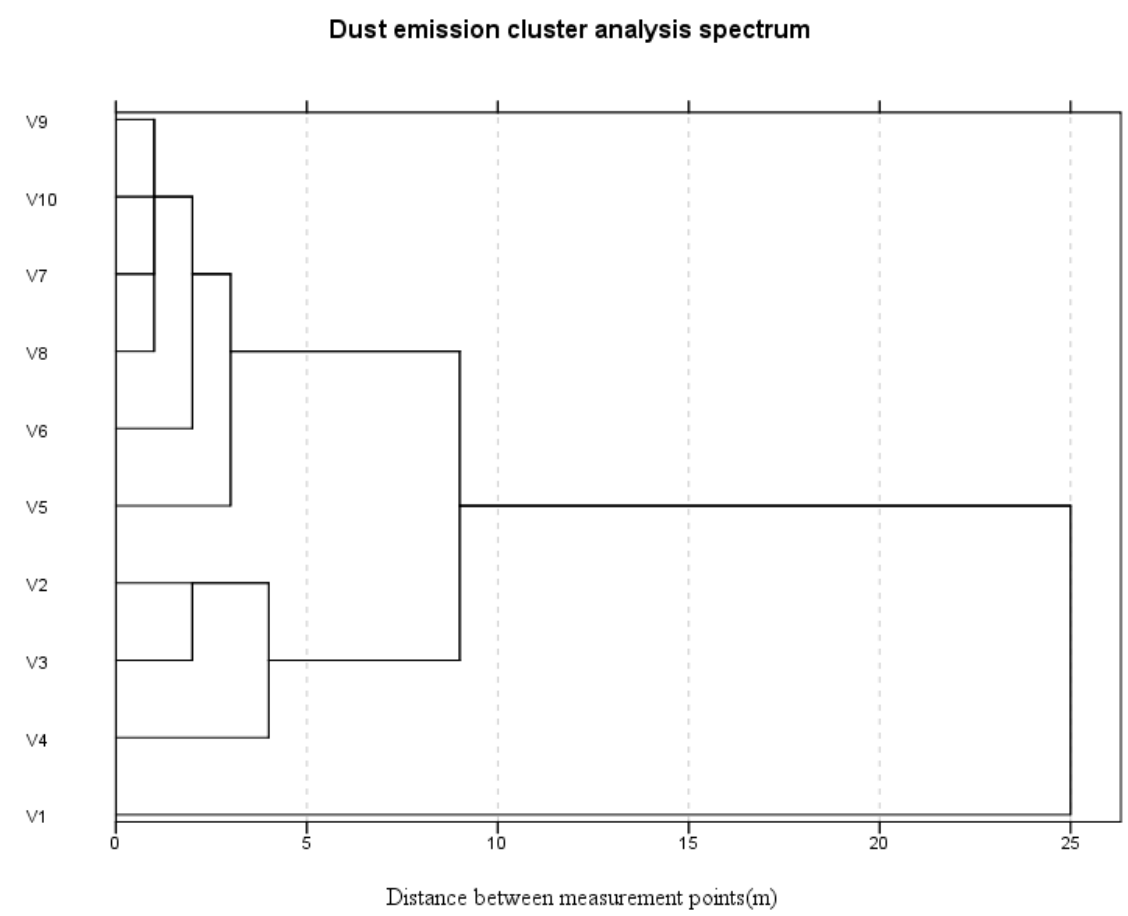

Figure 5. Clustering analysis spectrum. V1: the working channel passage; V2: excavator working area; V3: the hydraulic crushing hammer working area; V4: the construction waste dump; V5: loading vehicle; V6: the plane transport corridor; V7: the waste recycling area; V8: the road side; V9: living quarter of the workers; V10: out of the construction wall 


\section{Correlation between emission intensity and temperature and humidity}

Based on the classification result of system clustering, the correlation analysis of emission intensity, temperature and humidity for the location of each category is conducted. The analysis result is shown in Table 6.

Table 6. Correlation between emission strength and climate factor

\begin{tabular}{c|c|c|c}
\hline Factor & The first category & The second category & The third category \\
\hline Temperature & 0.296 & $0348^{* *}$ & 0.091 \\
Humidity & -0.353 & $-0.352^{* *}$ & -0.15 \\
\hline
\end{tabular}

** indicates the significant correction in 0.01

According to the results of the correlation analysis, it can be seen that only the emission intensity in the region of the first category is significantly correlated with temperature and humidity. The region of the second category has the largest area in the construction site, and the dust emission intensity is negatively correlated with humidity and positively correlated with temperature. A higher relative humidity in the air enables the particles in the construction site to combine into the larger particles with waters and gases, and promotes $\mathrm{PM}_{10}$ to be converted into larger particles, reducing the content of inhalable particles in the air, and decreasing the dust diffusion capacity, so as to decline the emission intensity index in the region of the second category. The emissions of the planar transport corridor, living quarter of the workers, roadside, waste collection area and loading vehicle in the region of the second category is mainly caused by the diffusion of particles.

As the area with the largest amount of dust emission on the construction site, the emission intensity in the region of the third category is mainly affected by the construction intensity, and the particles mainly come from themselves. It has a greater impact on the surrounding area and is less affected by temperature and humidity, so the correlation between emission intensity and these two factors is not significant. The emission intensity outside the wall is low, which is greatly affected by other factors such as the dust of the vehicle, thus reducing the correlation.

\section{Results}

It can be seen from Figure 6 that during the demolition process, the simulation result of the dust mass concentration is basically consistent with the measured data, and the distribution and variation laws are basically the same. The slight deviation between the two values is due to some errors in the field measurement, geometric model establishment and parameter setting process. Through comparative analysis, the movement, diffusion and settlement equations of demolition dust are verified, proving that it is feasible to use the discrete phase model to monitor and quantify the demolition construction dust distribution, and the simulation result is credible.

The region with the highest emission strength in the construction site is the operation channel, excavator working area, gunnery working area and construction dump. An independent sample $\mathrm{T}$ test is used to inspect the classification effect, a sound classification result has been obtained. 
In the region with the highest emission intensity, $\mathrm{PM}_{10}$ emission has a strong positive correlation with $\mathrm{PM}_{2.5}$ emission $\left(\mathrm{R}^{2}=0.817\right)$, namely, both pollutants have the same source of pollution.

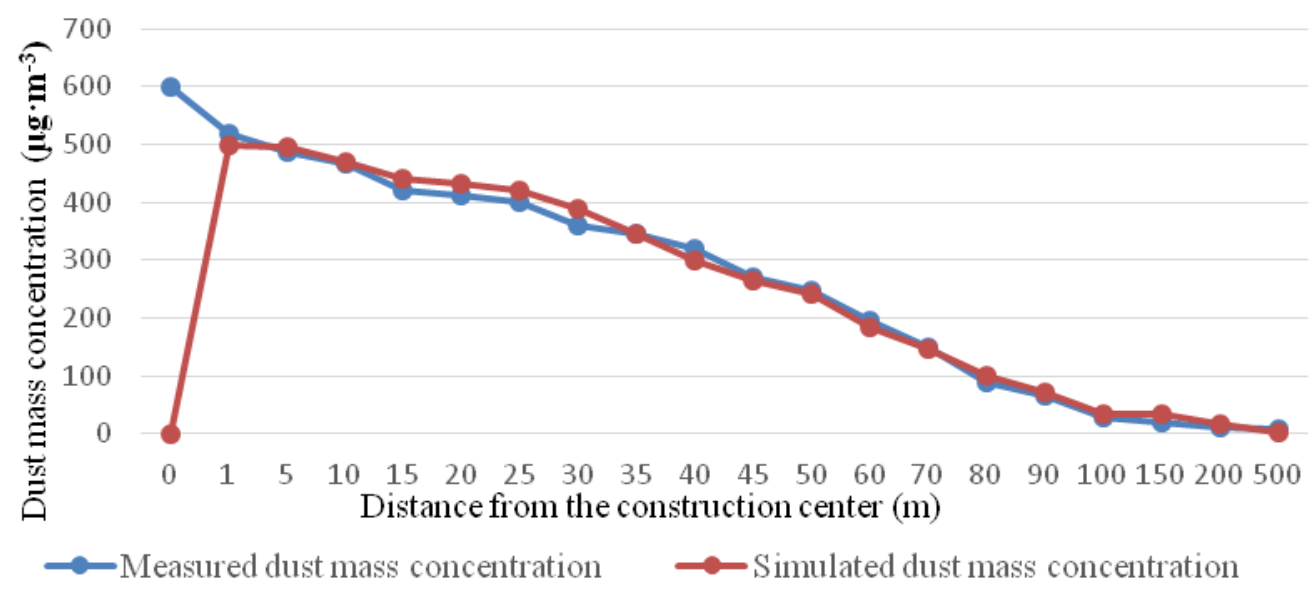

Figure 6. Comparative analysis of dust mass concentration simulation and actual measurement

\section{Discussion}

After analyzing the correlation between emission intensity and meteorological factors, it is concluded that the emission intensity of the plane transport corridor, living quarter of the workers, roadside, waste collection area and loading vehicle has a significant positive relationship with the temperature and a significant negative relationship with the humidity.

Moisture content of the dust: The higher the moisture content of the dust, the less likely the dust is to be raised.

Air temperature: When the temperature is low, the phenomenon of temperature inversion will probably occur, which hinders the convective movement of the air, so that the dust particles near the ground layer are not easily diffused, causing dust pollution. In the same condition, a lower temperature will lead to the probability of a higher dust concentration.

Air humidity: When the humidity of the air is high, the dust particles suspended in the air collide with the water molecules in the air, increasing the volume and mass of the dust particles, and enhancing the probability that the fine particles are mutually combined into large particles, thereby facilitating the sedimentation of dust particles.

\section{Conclusion}

Dust pollution during demolition operations creates a huge challenge for companies involved in such operations with regards to health and safety of workers and the neighboring community. By monitoring the change of dust concentration at the same height and at different distances near the boundary of the blasting site, this paper studied the dust diffusion law and formation law of the case study site in detail.

The main factors affecting the generation of construction dust include: (1) Moisture content of the dust. The higher the moisture content of the dust, the less likely the dust 
is to be raised. (2) Air temperature. When the temperature is low, the phenomenon of temperature inversion will probably occur, which hinders the convective movement of the air, so that the dust particles near the ground layer are not easily diffused, causing dust pollution. In the same condition, a lower temperature will lead to the probability of a higher dust concentration. (3) Air humidity. When the humidity of the air is high, the dust particles suspended in the air collide with the water molecules in the air, increasing the volume and mass of the dust particles, and enhancing the probability that the fine particles are mutually combined into large particles, thereby facilitating the sedimentation of dust particles.

Due to the limitations in the theory, there is no unified method or quantitative analysis possible to derive demolition intensity. Therefore, the influence of demolition intensity on dust emission intensity was not considered in this study. It could be a very useful further research as demolition intensity was found to be a deciding factor on dust generation. As a further research area, developing a quantitative tool to represent demolition intensity would facilitate an in-depth study of the relationship between the demolition method, its intensity and dust generation and diffusion.

Acknowledgements. This work is supported by A Value-added Research of EPC Project Based on Value Chain, Jiangxi Provincial Department of Education Science and Technology Project (GJJ180367); Spatial Effect, Realization Mechanism and Path of New Urbanization Inclusive Development in Underdeveloped Areas, Jiangxi Science and Technology Plan Project (20181BAA208017).

\section{REFERENCES}

[1] Alekhfne, F. (2012): Gas-particles flow transitions for high density powder. - Lecture Notes in Engineering and Computer Science 1: 1820-1825.

[2] Ansart, R., Ryck, A., Dodds, J. A., Roudet, M., Fabre, D., Charru, F. (2009): Dust emission by powder handling: comparison between numerical analysis and experimental results. - Powder Technology 190(1-2): 274-281.

[3] Cerecedo, L. M., Aisa, L., Ballester, J. (2009): Experimental study on a non-dilute twophase coflowing jet: Dynamics of particles in the near flow field. - International Journal of Multiphase Flow 35(5): 468-483.

[4] Chen, B., Chen, Z., Deng, L. (2016): Building change detection with RGB-D map generated from UAV images. - Neuro Computing 208: 350-364.

[5] Chen, J. S., Jiang. L., Jiang, Z. G. (2015): Numerical simulation of dust distribution and its influencing factors in slope drilling. - Journal of Engineering Science 37(6): 685-692.

[6] Chou, J. S., Yeh, K. C. (2016): Life cycle carbon dioxide emissions simulation and environmental cost analysis for building construction. - Journal of Cleaner Production 101: 137-147.

[7] Dong, S. H., Xie, Y., Huang, F. Y. Q., Shi, X. R., Yi, R., Shi, G. L., Feng, Y. (2019): Assessment of heavy metal sources and potential health risks of $\mathrm{PM}_{2.5}$ in Yangzhou. Environmental Science 2: 1-10.

[8] Fan, S. B., Yang, T., Wang, K., Li, X. F. (2019): Establishment method and application of road raise dust emission factor establishment. - Environmental Study 4: 1-13.

[9] Huang, Y., Qu, S., Song, G., Li, G., Tian, G. (2013): Comparison of dust source particle composition screening method. - Environmental Science Research 26(5): 522-526.

[10] Kohlman, R. E. R., Shapira, A., Martins, A. R. B. (2014): Characterization and evaluation dust on building construction sites in Brazil. - Neurochemistry International 5(1): 1-8.

[11] Li, M., Wu, C. (2006): Visualization of dust point pollution diffusion model. Environmental Science and Technology 11(12-14): 115-116. 
[12] Li, X. D., Su, S., Huang, T. J. (2014): Monitoring and analysis of raise dust of the building in secondary structure and indoor rough decoration stages. - Journal of Chinese Safety Sciences 24(8): 103-106.

[13] Li, X. D., Su, S., Huang, T. J. (2015): Quantitative evaluation of health damage caused by construction dust. - Journal of Tsinghua University (Natural Science Edition) 55(1): 5055.

[14] Ma, G. X., Han, Y., Lu, J. F., Yao, J., You, S. D. (2018): Design and achievement of automatic monitoring system for dust pollution sources based on UAV. - Environmental Monitoring in China 34(1): 151-156.

[15] Madelyn, M., Antonio, R. D. A. (2010): The building cost system in Andalusia: application to construction and demolition waste management. - Construction Management and Economics 28(5): 495-507.

[16] Mohamed, M., Magdy, M., Azza, A. Z., Ei-said, M. (2008): Handling construction pollutions using multi-objective optimization. - Construction Management and Economics 26(10): 1113-1125.

[17] Muleski, G. E., Cowherd, C. J., Kinsey, J. S. (2005): Particulate emission from construction activities. - Journal of the Air \& Waste Management Association 55: 772783.

[18] Peng, X., Shi, G., Liu, G., Xu, J., Tian, Y., Zhang, Y., Feng, Y., Russell, A. G. (2016): Source apportionment and heavy metal health risk (HMHR) quantification from sources in a southern city in China, using an ME2-HMHR model. - Environmental Pollution 221: 335-342.

[19] Shi, P., Du, K., Mu, C., Wang, K., Wang, Y. (2013): Vertical scanning radar observation method for unorganized raise dust of the construction site. - Optical Journal 33(3): 39-45.

[20] Sun, Z. Q., Fang, B. J. (2016): Analysis of dust stress and its movement in construction tunnel. - Coal Technology 35(5): 176-178.

[21] Sung, K. P., Liu, C. L., Craig, L. (2006): Case study of demolition costs of residential buildings. - Construction Management and Economics 24(9): 967-976.

[22] Thomas, L. D., Hodgson, S., Nieuwenhuijsen, M., Jarup, L. (2009): Early kidney damage in a population exposed to cadmium and other heavy metals. - Environmental Health Perspectives 117(2): 181-184.

[23] Toropov, E. V., Osintsev, K. V., Aliukov, S. V. (2018): Analysis of the calculated and experimental dependencies of the combustion of coal dust on the basis of a new methodological base of theoretical studies of heat exchange processes. - International Journal of Heat and Technology 36(4): 1240-1248.

[24] Wang, R., Chen, B. (2014): Three-dimensional lattice vortex method for simulating free falling particle groups. - Engineering Thermophysics 35(3): 499-502.

[25] Wu, F. B., Zhang, H. K., Lv, Q. T. (2017): Design of environmental monitoring system based on embedded construction site. - Modern Electronic Technique 40(20): 72-76.

[26] Wu, Z., Zhang, X., Wu, M. (2015): Mitigating construction dust pollution: state of the art and the way forward. - Journal of Cleaner Production 112: 1658-1666.

[27] Xiao, H., Yang, X., Wu, Q., Bai, Q., Chen, H., Chen, X., Xue, R., Du, M., Huang, L., Wang, R., Wang, H. (2019): Estimate emissions of construction fugitive dust in Xi'an. Chinese Journal of Environmental Science 1: 222-228.

[28] Zhi, X., Jiang, Z., Tang, M., Jiang, X (2018): Force analysis of settling dust on dust collecting plate and secondary flying research. - Coal Journal 33(3): 310-313.

[29] Zhu, P., Zhang, C., Li, D. M., Bai, S. (2017): Research on numerical simulation of dust distribution in belt conveyer lane. - Journal of North China Institute of Science and Technology 14(6): 40-46.

[30] Zuo, J., Raufdeen, R., Matthew, H. (2017): Dust pollution control on construction site: awareness and self-responsibility of managers. - Journal of Cleaner Production 166: 312320 . 